\title{
Daytime Mouthpiece for Continuous Noninvasive Ventilation in Individuals With Amyotrophic Lateral Sclerosis
}

\author{
Marie-Eve Bédard MD FRCPC and Douglas A McKim MD FRCPC DABSM
}

\begin{abstract}
BACKGROUND: Noninvasive ventilation (NIV) is commonly used to provide ventilatory support for individuals with amyotrophic lateral sclerosis (ALS). Once 24-h ventilation is required, the decision between invasive tracheostomy ventilation and palliation is often faced. This study describes the use and outcomes of daytime mouthpiece ventilation added to nighttime mask ventilation for continuous NIV in subjects with ALS as an effective alternative. METHODS: This was a retrospective study of 39 subjects with ALS using daytime mouthpiece ventilation over a 17-y period. RESULTS: Thirty-one subjects were successful with mouthpiece ventilation, 2 were excluded, 2 stopped because of lack of motivation, and 4 with bulbar subscores of the Revised Amyotrophic Lateral Sclerosis Functional Rating Scale (b-ALSFRS-R) between 0 and 3 physically failed to use it consistently. No subject in the successful group had a b-ALSFRS-R score of $<6$. Thirty of the successful subjects were able to generate a maximum insufflation capacity - vital capacity difference with lung volume recruitment. The median (range) survival to tracheostomy or death from initiation of nocturnal NIV and mouthpiece ventilation were $648(176-2,188)$ and 286 (41-1,769) d, respectively. Peak cough flow with lung-volume recruitment $>180 \mathrm{~L} / \mathrm{min}$ at initiation of mouthpiece ventilation was associated with a longer survival $(637 \pm 468 \mathrm{vs} 240 \pm 158 \mathrm{~d}(P=.01)$. CONCLUSIONS: Mouthpiece ventilation provides effective ventilation and prolonged survival for individuals with ALS requiring full-time ventilatory support and maintaining adequate bulbar function. Key words: mouthpiece ventilation; noninvasive ventilation; amyotrophic lateral sclerosis; NIV; ALS. [Respir Care 2016;61(10):1341-1348. () 2016 Daedalus Enterprises]
\end{abstract}

\section{Introduction}

Amyotrophic lateral sclerosis (ALS) is an incurable neurodegenerative disease affecting upper and lower motor neurons and resulting in progressive skeletal muscle weakness. Respiratory failure is the most common cause of death, and is often associated with pneumonia. ${ }^{1}$ The median survival after symptom onset is $3 \mathrm{y}^{2}$

There is evidence for the benefit of nocturnal noninvasive ventilation (NIV) in ALS. One randomized controlled

\footnotetext{
The authors are affiliated with the Canadian Alternatives in Noninvasive Ventilation (CANVent) Program, Ottawa Hospital Rehabilitation Centre, Division of Respirology, Department of Medicine, University of Ottawa, Ottawa, Ontario, Canada.

The authors have disclosed no conflicts of interest.

Correspondence: Douglas A McKim MD FRCPC DABSM, CANVent Program, Ottawa Hospital Rehabilitation Centre, 505, Smyth Road, Ottawa, Ontario K1H 8M2, Canada. E-mail: dmckim@ottawahospital.on.ca.
}

DOI: $10.4187 /$ respcare.04309 trial demonstrated improved survival and quality of life with the use of NIV in ALS subjects without severe bulbar dysfunction, and improved quality of life in those with poor bulbar function. ${ }^{3}$ Improved survival and quality of life were also reported by several non-randomized studies as were improved gas exchange, reduced rate of decline in vital capacity (VC), and enhanced cognition. -15 $^{-15}$

Although NIV is initiated during sleep, continuous, 24-h ventilation may eventually be required, and invasive tracheostomy ventilation is often discussed. Although potentially necessary for extended survival, tracheostomy is associated with long-term morbidity, a high burden of care, and frequent residence in an acute or chronic care facility. ${ }^{16}$

Subjects with sufficient bulbar muscle function to hold an insufflation deeper than their spontaneous VC with lungvolume recruitment and to generate assisted peak cough flow $>180 \mathrm{~L} / \mathrm{min}$ have demonstrated successful use of 24-h NIV, regardless of their VC or hours of ventilatory support needed. ${ }^{17}$ Continuous use of conventional mask interfaces limits speech, nutrition, and mobility and can 


\section{Daytime Mouthpiece NIV in Subjects With ALS}

cause skin breakdown. Daytime ventilation with volumetargeted mouthpiece ventilation eliminates these issues.

To our knowledge, publication on mouthpiece ventilation in ALS is limited to 19 subjects in a total of 3 studies. ${ }^{17-19}$ Few details were provided about its use, and some subjects may have used orally retained interfaces.

The primary objective of our study was to describe the use and outcomes of daytime mouthpiece ventilation in addition to nocturnal conventional mask NIV for 24-h ventilation in a subset of individuals with ALS with relatively preserved bulbar function. The secondary objective was to assess factors contributing to its successful use.

\section{Methods}

\section{Study Population}

Study subjects were from the Ottawa Hospital Rehabilitation Centre's interprofessional ALS clinic/Canadian Alternatives in Noninvasive Ventilation (CANVent) Program. Subjects were all referred by neurologists and diagnosed with definite or probable ALS using El Escorial criteria. ${ }^{20}$

Subjects completed respiratory assessments and pulmonary function testing every 2-6 months, depending on the rate of progression. Testing included measurement of FVC, maximum inspiratory pressure, maximum expiratory pressure, maximum voluntary ventilation, and maximum insufflation capacity. Predicted values were from the National Health and Nutrition Examination Survey III. ${ }^{21}$ The maximum insufflation capacity is the volume of air exhaled from a maximum lung inflation obtained with lungvolume recruitment. ${ }^{22}$ The latter was done with a handheld resuscitation bag via a mouthpiece or face mask. By closing the glottis and retaining each inspiratory volume, the patient stacks multiple breaths, reaching a lung volume closer to normal predicted. Spontaneous peak cough flow and assisted peak cough flow immediately after lung-volume recruitment were measured using a peak flow meter with the subject forcibly coughing into a mouthpiece or face mask. We have used the Revised Amyotrophic Lateral Sclerosis Functional Rating Scale (ALSFRS-R), ${ }^{23}$ a validated questionnaire for measuring physical function, since 2000. It includes 12 items assessing bulbar, limb, and respiratory function, each rated on a 5-point scale from 4 (normal function) to 0 (maximum impairment). A bulbar subscale score (bALSFRS-R) is recorded from the first 3 items (speech, salivation, and swallowing) and scored from 0 to $12 .{ }^{24}$

When VC falls below $80 \%$ of predicted and/or peak cough flow is $<270 \mathrm{~L} / \mathrm{min}$, lung-volume recruitment at least twice daily is prescribed. If the peak cough flow with lung-volume recruitment remains $<270 \mathrm{~L} / \mathrm{min}$, manually assisted cough and/or mechanical in-exsufflation, if available, is introduced..$^{25}$

\section{QUICK LOOK}

\section{Current knowledge}

Noninvasive ventilation improves survival and quality of life of patients with amyotrophic lateral sclerosis (ALS). It is generally provided through mask ventilation. Once 24-h ventilation is required, patients often face the choice of tracheostomy or palliation. Mouthpiece ventilation is a possible alternative.

\section{What this paper contributes to our knowledge}

Mouthpiece ventilation is a safe, convenient, and effective way of providing noninvasive ventilation in ALS individuals with preserved bulbar function. Survival of those who achieved peak cough flows $>180 \mathrm{~L} / \mathrm{min}$ was significantly longer. A Revised ALS Functional Rating Scale bulbar subscale score of $\geq 6$ could be a predictor of success.

Patient education included a session on respiratory care, NIV, and advance directives. ${ }^{26}$ Indications for nocturnal NIV included orthopnea, daytime hypercapnia, symptoms of sleep-disordered breathing, FVC $<50 \%$ of predicted, or maximum inspiratory pressure $<40 \mathrm{~cm} \mathrm{H}_{2} \mathrm{O} .{ }^{27,28}$ Nocturnal bi-level parameters were determined in the out-patient clinic. Subsequent adjustments were made based on comfort, symptoms, downloaded bi-level data, ${ }^{29}$ carbon dioxide level, and overnight oximetry.

With progression of respiratory muscle weakness, patients will increase use of NIV to control hypercapnia and/or relieve daytime dyspnea. When NIV use is $>12 \mathrm{~h} /$ day, mouthpiece ventilation is recommended for those who wish to pursue 24-h NIV and who maintain sufficient bulbar function to retain a mouthpiece and achieve an adequate seal around it in order to maintain adequate ventilation and perform lung-volume recruitment.

For initiation of mouthpiece ventilation, an out-patient education session with a trial and adjustment of ventilator parameters was provided. For the successful subject, 2 volume-targeted devices are ordered. On arrival, the first ventilator tubing and mouthpiece are mounted on the wheelchair (Fig. 1), and the settings are adjusted. The continuous mandatory ventilation mode is used. Tidal volume (from 800 to $1,800 \mathrm{~mL}$ ), inspiratory time, and breathing frequency were set according to the subject's need and comfort. The second ventilator is used in pressure-control mode with previous nighttime parameters and replaces the bi-level device.

\section{Study Design}

A retrospective chart review was performed of every subject for whom mouthpiece ventilation was prescribed 


\section{Daytime Mouthpiece NIV in Subjects With ALS}

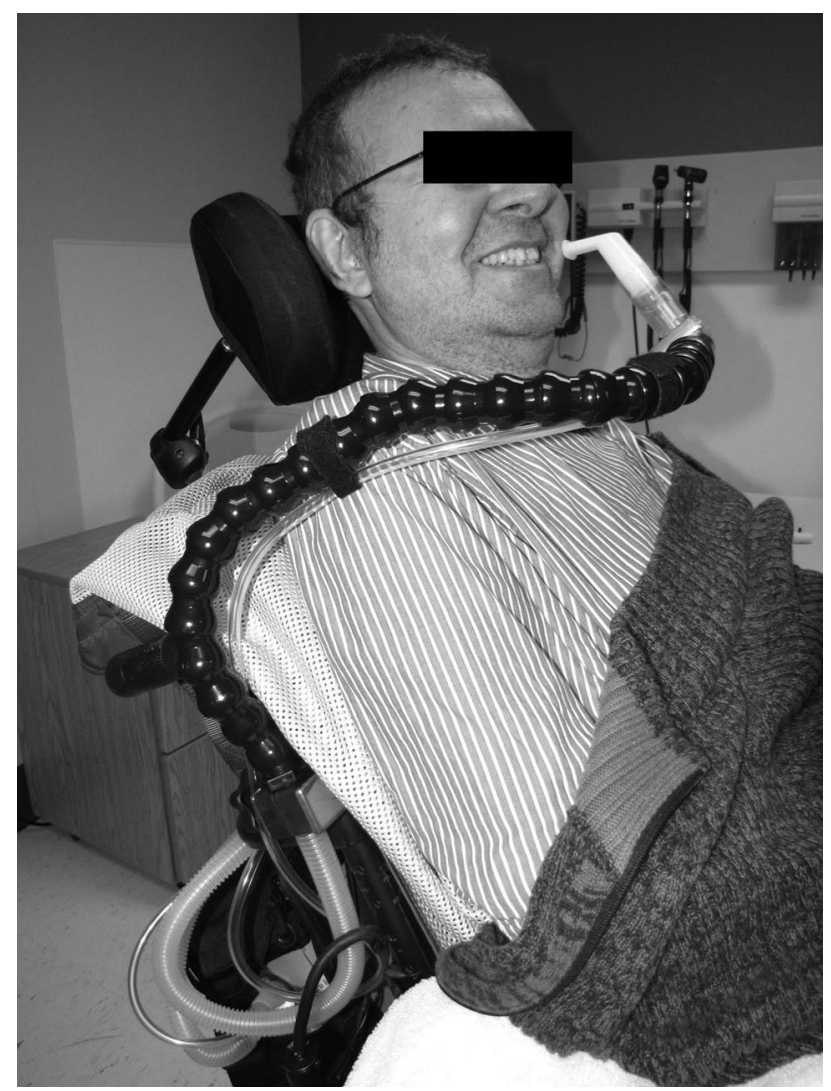

Fig. 1. Subject using mouthpiece noninvasive ventilation.

from January 1996 to December 2012. Demographic data, age at onset of symptoms, and age at diagnosis were recorded. Dates were recorded for symptom onset, diagnosis, introduction of nocturnal NIV, NIV utilization $>12 \mathrm{~h} / \mathrm{d}$, initiation of mouthpiece ventilation, and tracheostomy or death. All pulmonary function test scores and ALSFRS-R and $\mathrm{b}$-ALSFRS-R scores were reviewed, and the nearest values to each of the timeline dates were recorded. Time from diagnosis to initiation of nocturnal NIV was calculated, and indications for introduction of nocturnal NIV were noted. Time from mouthpiece ventilation to tracheostomy or death was determined as well as time to tracheostomy or death from symptom onset, diagnosis, and initiation of nocturnal NIV. Successful use of mouthpiece ventilation was defined as consistent use for at least 1 month. The study was approved by the Ottawa Health Science Network Research Ethics Board (2007951-O1H).

\section{Statistical Analysis}

Statistical analyses were carried out using SAS 9.2 (SAS Institute, Cary, North Carolina). Results are expressed as mean \pm SD unless otherwise specified. The Wilcoxon test was used for comparison of ALSFRS-R and b-ALSFRS-R between groups with significance level set at $P<.05$.
Table 1. Characteristics of Subjects on Initial Assessment

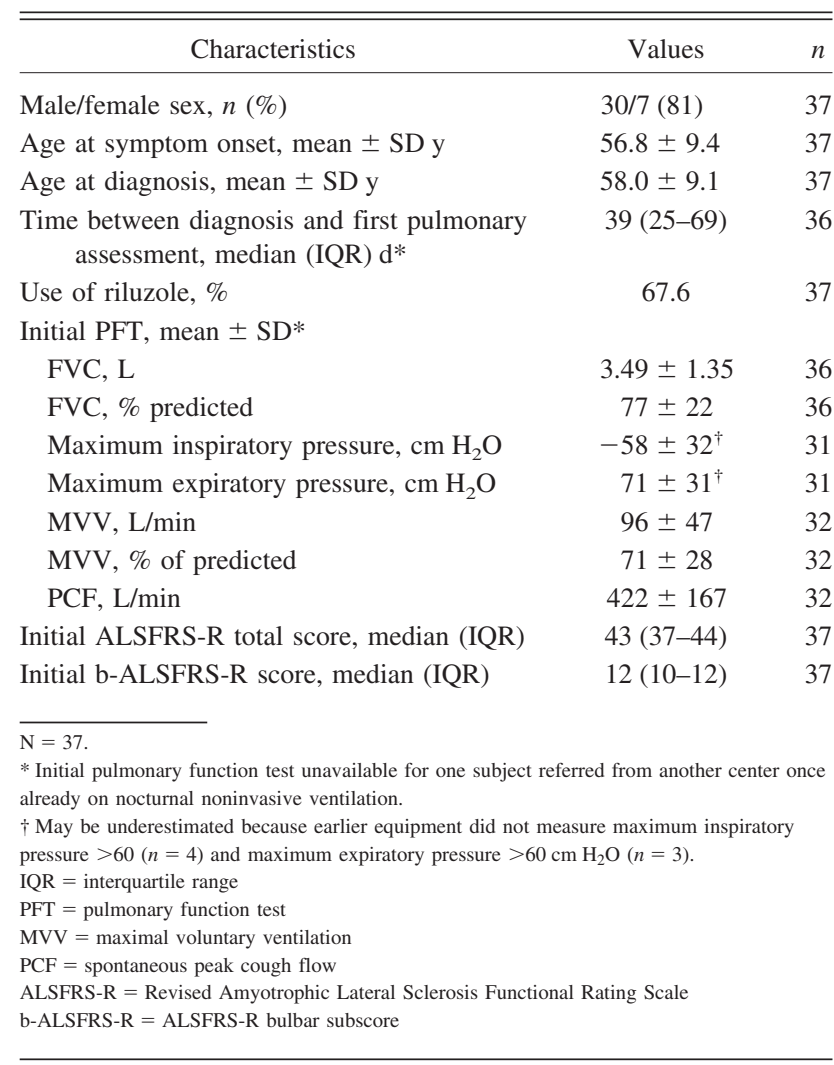

Analysis of survival was undertaken using the KaplanMeier method, and the log-rank test was applied for between-group comparison of survival.

\section{Results}

During the study period, 204 patients were prescribed NIV. Thirty-nine of these (19\%) were prescribed mouthpiece ventilation. The first 2 subjects successfully used mouthpiece ventilation for 770 and $852 \mathrm{~d}$ but were excluded from the analysis due to limited data.

Thirty-seven subjects were included in the analysis. Baseline characteristics are presented in Table 1. Time from diagnosis to initiation of nocturnal NIV varied from $-43 \mathrm{~d}$ (one subject started before diagnosis) to $1,541 \mathrm{~d}$, with a median of $317 \mathrm{~d}$. Twenty-six subjects $(70 \%)$ had more than one indication for nocturnal NIV. FVC $<50 \%$ of predicted was the most common indication ( 25 of 37 ), followed by maximum inspiratory pressure $<40 \mathrm{~cm} \mathrm{H}_{2} \mathrm{O}$ (22 of 37), daytime hypercapnia (16 of 37), symptomatic sleep-disordered breathing (10 of 37), orthopnea ( 8 of 37), and finally acute respiratory failure (5 of 37).

Time from introduction of nocturnal NIV to initiation of mouthpiece ventilation ranged from 0 to $852 \mathrm{~d}$, with a median of $200 \mathrm{~d}$. Five began mouthpiece ventilation si- 


\section{Daytime Mouthpiece NIV in Subjects With ALS}

Table 2. Characteristics of Successful Subjects at Time of Initiation of Mouthpiece Ventilation

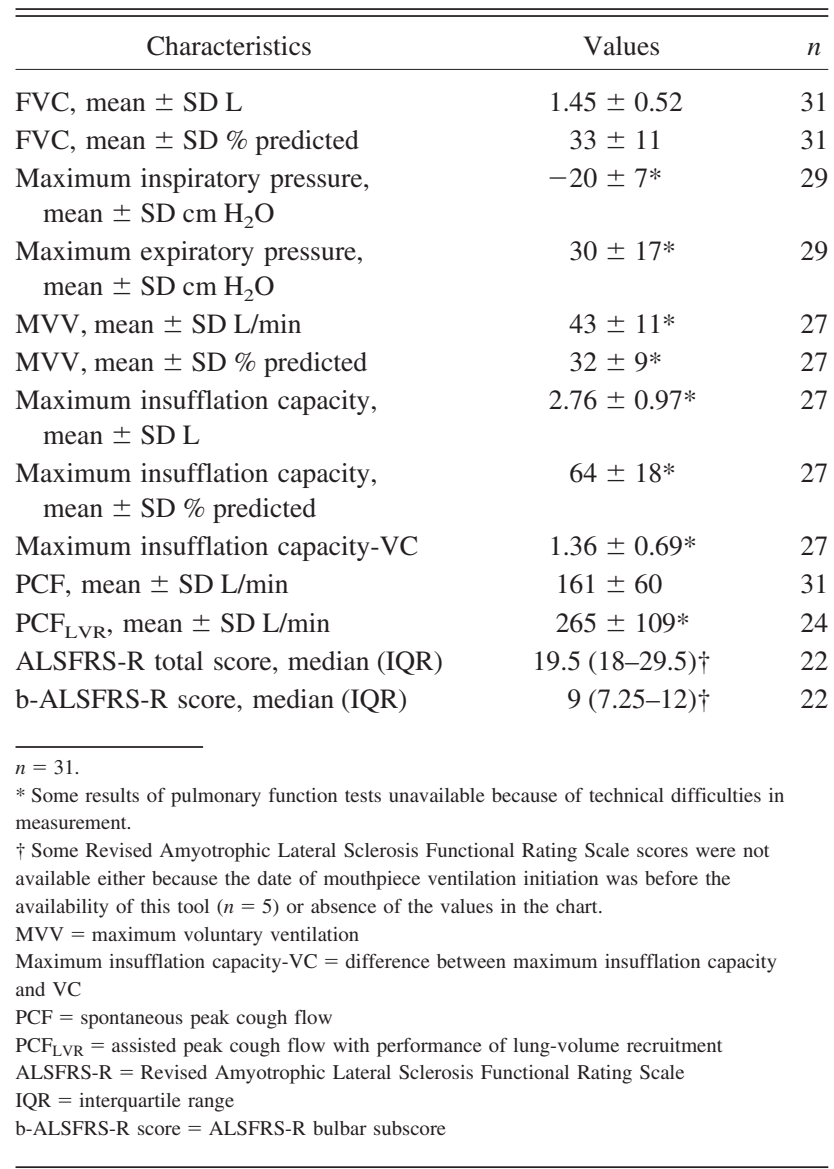

multaneously with nocturnal NIV: 2 were extubated after acute respiratory failure directly to nocturnal NIV and daytime mouthpiece ventilation, 2 had daytime dyspnea and hypercapnia, and one had important daytime dyspnea and a rapidly declining FVC.

For 24 of the remaining 32 subjects, downloaded bilevel data and/or clinical notes allowed the identification of daily use of NIV $>12 \mathrm{~h}$ before the initiation of mouthpiece ventilation. Information was unavailable for 3 subjects, and 5 had mouthpiece ventilation introduced at $<12 \mathrm{~h}$ of daily NIV use because of significant daytime shortness of breath insufficiently relieved by NIV or intolerance of mask NIV while awake. In all subjects, daytime hypercapnia or symptoms of dyspnea were resolved with the addition of mouthpiece ventilation.

Six of the 37 subjects used mouthpiece ventilation for $<1$ month and were deemed unsuccessful subjects. Review of their charts identified 2 groups. Two subjects, although fully capable of mouthpiece ventilation, chose not to use it, preferring to use mask NIV to alleviate their symptoms. They had b-ALSFRS-R scores of 10 and 12, generated maximum insufflation capacity-VC differences
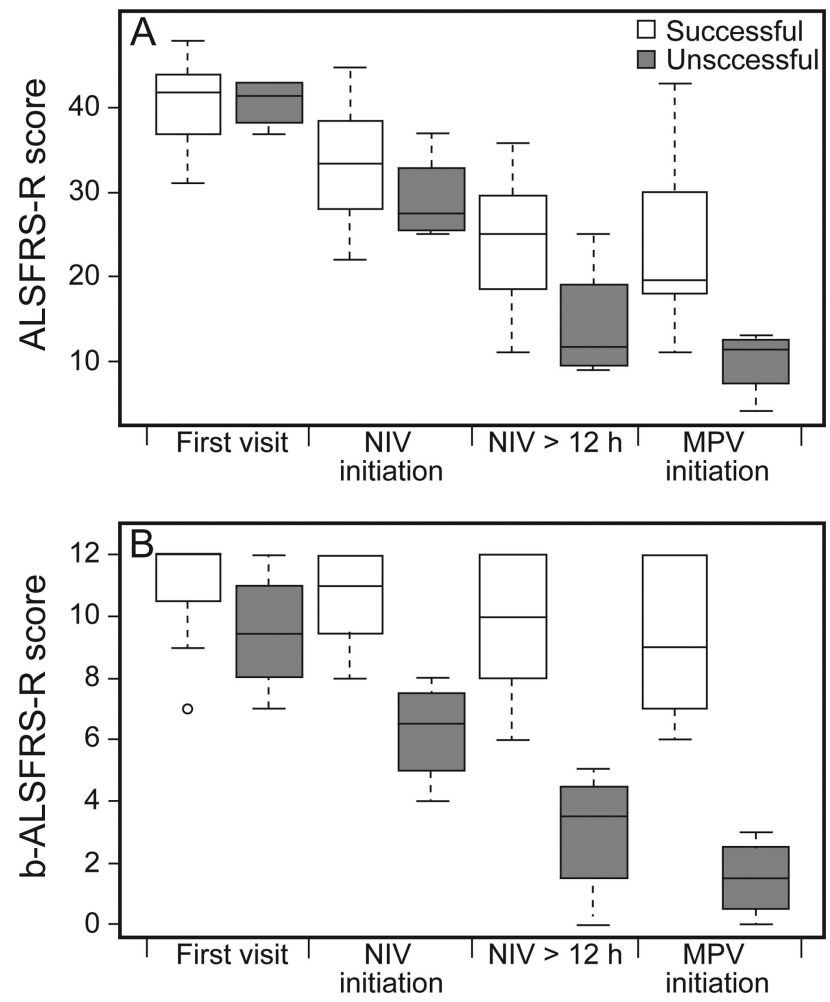

Fig. 2. Evolution of Revised Amyotrophic Lateral Sclerosis Functional Rating Scale scores over time in subjects successful and unsuccessful with mouthpiece ventilation (MPV). The center lines represent the median value, boxes span the first quartile to the third quartile, and whiskers denote minimum and maximum values that are within $1.5 \times$ the interquartile range. Circles are outliers. ALSFRS-R = Revised Amyotrophic Lateral Sclerosis Functional Rating Scale (0-48), b-ALSFRS-R = ALSFRS-R bulbar sub-score $(0-12)$, NIV $=$ noninvasive ventilation.

of 2.76 and $1.51 \mathrm{~L}$, and had peak cough flow with lungvolume recruitment of 380 and $460 \mathrm{~L} / \mathrm{min}$, respectively. The remaining 4 were highly motivated but physically failed to use it adequately. They each had a b-ALSFRS-R score between 0 and 3, only one achieved a maximum insufflation capacity-VC difference, only one had a peak cough flow $>180 \mathrm{~L} / \mathrm{min}$, and none demonstrated an increase in peak cough flow with lung-volume recruitment.

Characteristics of the 31 successful subjects at initiation of mouthpiece ventilation are presented in Table 2. Of the 27 subjects with available maximum insufflation capacity values, all but one demonstrated a maximum insufflation capacity-VC difference. Of the 24 subjects with available peak cough flow with lung-volume recruitment values, only 7 were $<180 \mathrm{~L} / \mathrm{min}$. The b-ALSFRS-R scores (available for 22 subjects) were significantly higher than for the 4 unsuccessful subjects, with median (interquartile range) of $9(7.25-12)$ versus $1.5(0.75-2.25)$, respectively $(P=.002)$. Moreover, no subject in the successful group had a b-ALSFRS-R $<6$. The values of the ALSFRS-R scores over time in the 2 groups are shown in Figure 2. 


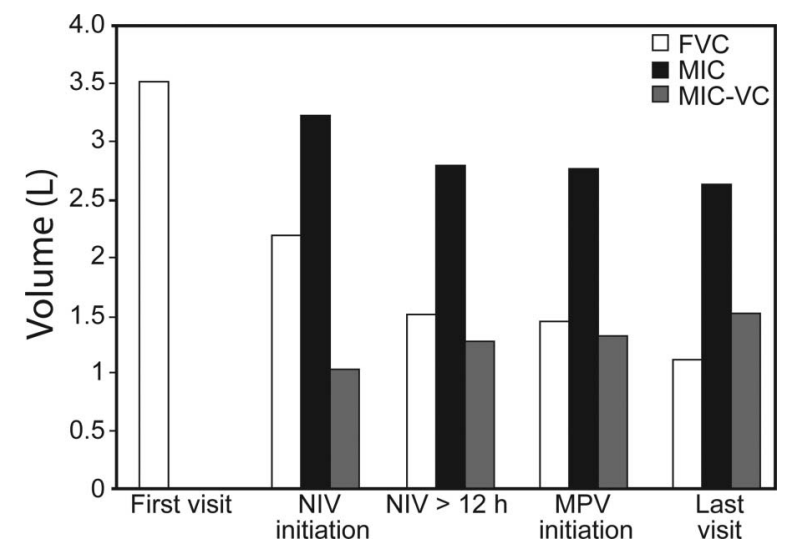

Fig. 3. Evolution of pulmonary function testing over time in subjects successful with mouthpiece ventilation (MPV). Maximum insufflation capacity (MIC) is not shown at the first visit because it was only done when FVC was $<80 \%$ of predicted. NIV $=$ noninvasive ventilation. MIC-VC = difference between MIC and vital capacity.

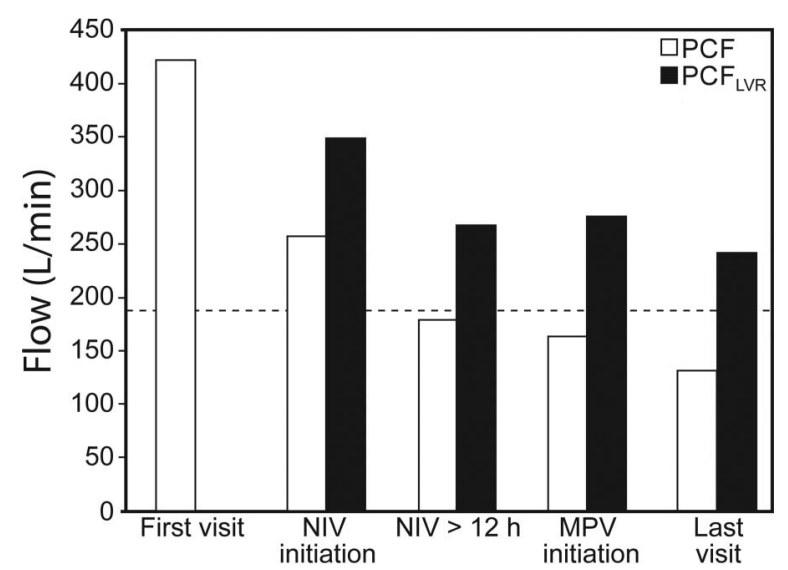

Fig. 4. Evolution of spontaneous and assisted peak cough flow (PCF) over time in subjects successful with mouthpiece ventilation (MPV). The dotted line shows $180 \mathrm{~L} /$ min assisted peak cough flow with performance of lung volume recruitment. NIV = noninvasive ventilation. $\mathrm{PCF}_{\mathrm{LVR}}=$ assisted $\mathrm{PCF}$ with performance of lung volume recruitment.

The small number of unsuccessful subjects precluded performance of multivariate regression analysis of factors associated with successful mouthpiece ventilation.

The evolution of FVC, maximum insufflation capacity, and maximum insufflation capacity-VC difference as well as peak cough flow and peak cough flow with lung-volume recruitment in successful subjects are shown in Figures 3 and 4. The FVC fell over time, whereas the maximum insufflation capacity remained relatively well preserved, and the maximum insufflation capacity-VC difference increased. Peak cough flow and, to a lesser degree, peak cough flow with lung-volume recruitment both decreased, but the latter remained $>180 \mathrm{~L} / \mathrm{min}$.
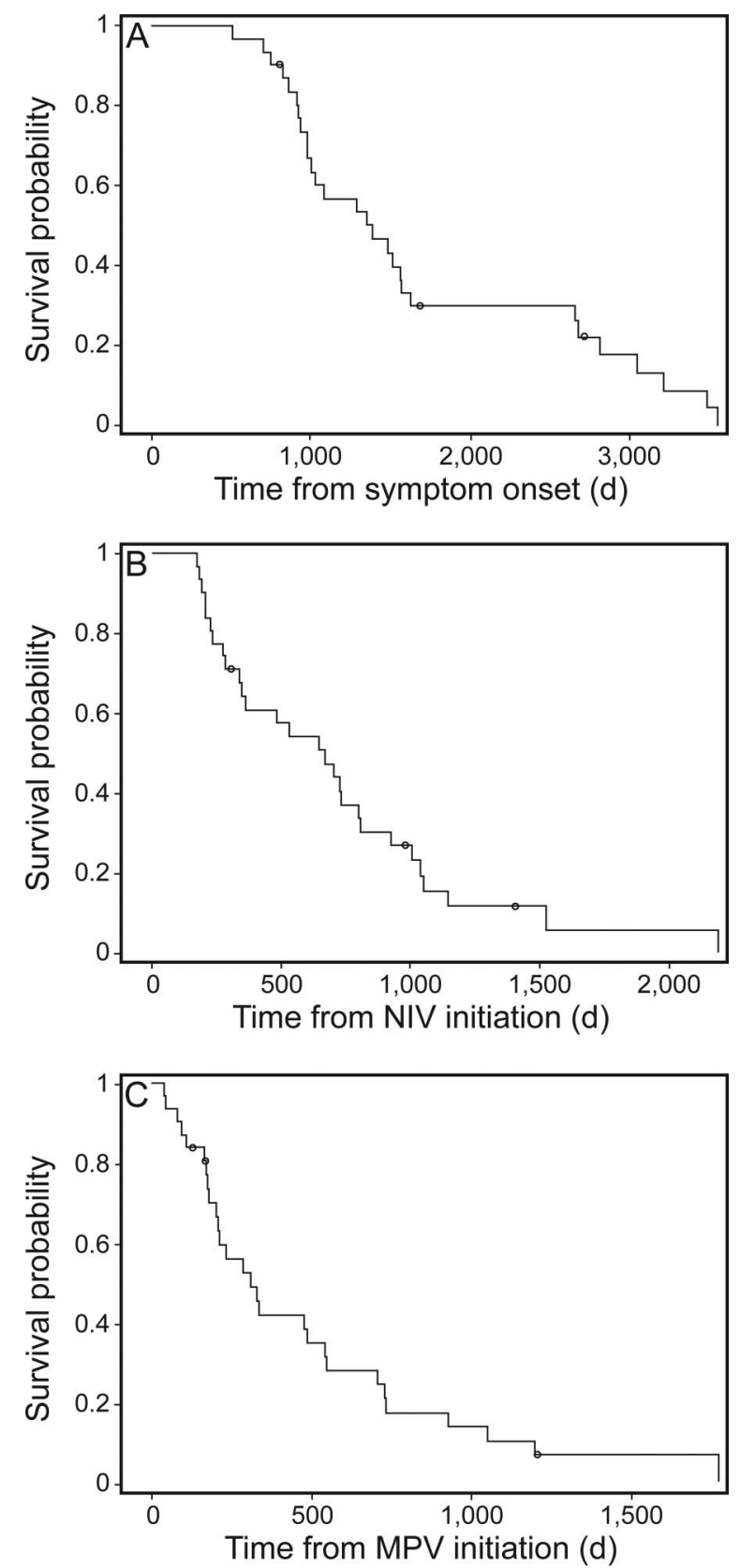

Fig. 5. Kaplan-Meier curves of subjects successful with mouthpiece ventilation from symptom onset $(A)$, initiation of noninvasive ventilation (NIV) $(B)$, and introduction of mouthpiece ventilation (MPV) to death or tracheostomy (C). Points denote subjects still alive.

Five subjects went on to tracheostomy, 4 in the context of acute respiratory failure and one electively. Three subjects stopped using mouthpiece ventilation after 82, 197, and $372 \mathrm{~d}$ because of a preference for mask-NIV or an inability to seal around the mouthpiece because of progressive bulbar impairment.

The Kaplan-Meier curves for successful subjects are shown in Figure 5. Median (range) survival to tracheos- 


\section{Daytime Mouthpiece NIV in Subjects With ALS}

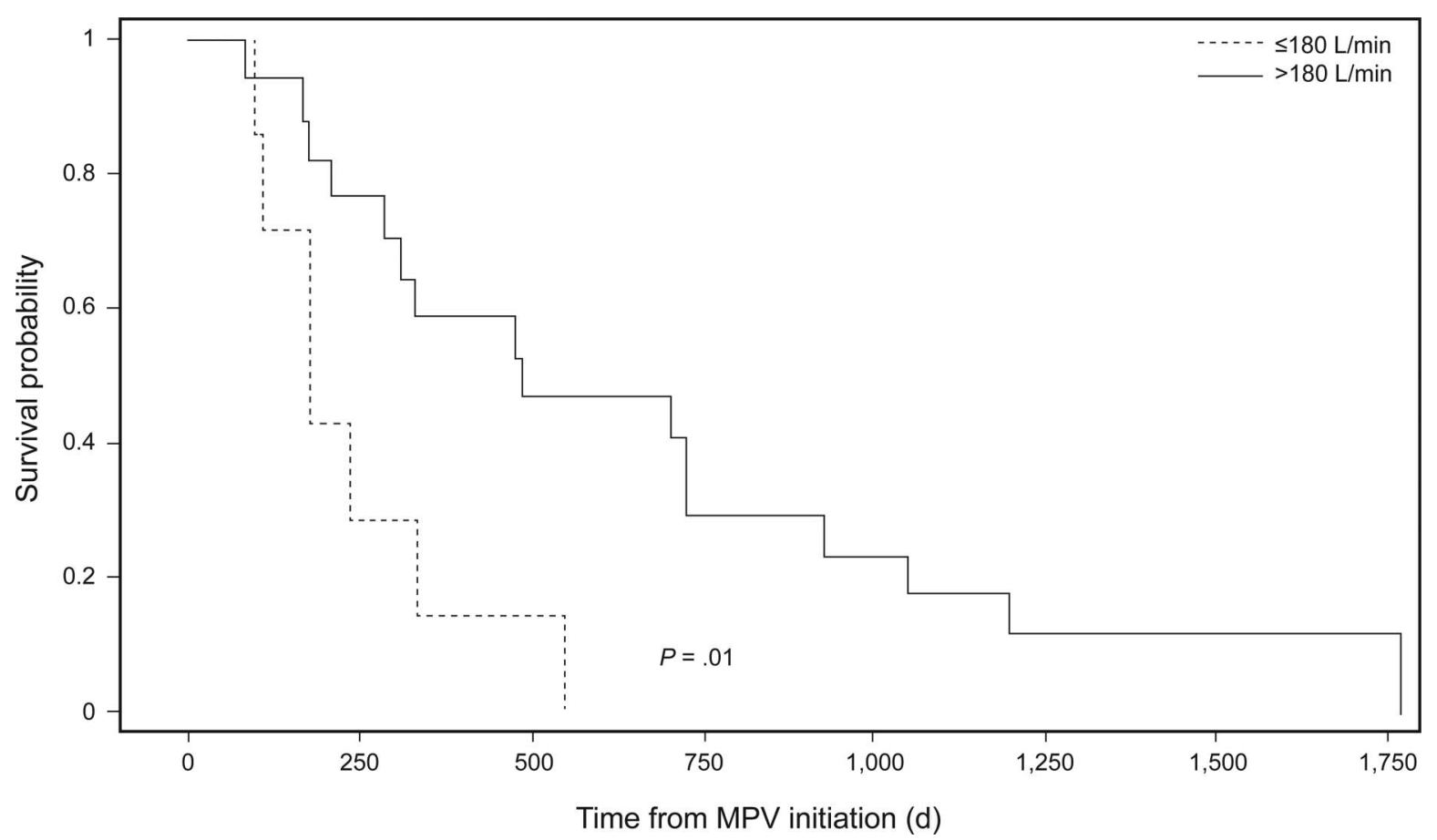

Fig. 6. Kaplan-Meier curves of subjects successful with mouthpiece ventilation (MPV) according to assisted peak cough flow (with lung volume recruitment) $>180 \mathrm{~L} / \mathrm{min}$ versus $\leq 180 \mathrm{~L} / \mathrm{min}$.

tomy or death from mouthpiece ventilation initiation, initiation of nocturnal NIV, and symptom onset were 286 (41-1,769), 648 (176-2,188), and 1,353 (512-3,555)d, respectively. Three subjects are still alive. In those for whom values were available, a peak cough flow with lungvolume recruitment $>180 \mathrm{~L} / \mathrm{min}$ at initiation of mouthpiece ventilation was associated with longer survival with mouthpiece ventilation $(637 \pm 468 \mathrm{~d}$ vs $240 \pm 158 \mathrm{~d}$ $[P=.01]$ ) (Fig. 6). No value associated with significantly improved survival was identified for maximum insufflation capacity-VC difference, FVC ( $\mathrm{L}$ or percentage of predicted), or b-ALSFRS-R score.

Of the 27 successful mouthpiece ventilation users who did not undergo tracheostomy, only 2 were placed in a chronic care facility. Of the 24 deceased, 17 died at home.

\section{Discussion}

This study is the first to fully describe utilization of daytime mouthpiece ventilation in an ALS population using 24-h NIV. Results confirm the effectiveness of mouthpiece ventilation as well as the importance of preserved bulbar function and ability to generate a maximum insufflation capacity-VC difference and adequate peak cough flow with lung-volume recruitment for survival. Use of the b-ALSFRS-R score in this context is described for the first time.

Despite its effectiveness and convenience, mouthpiece ventilation is seldom used in individuals with ALS need- ing continuous ventilatory support. Tracheostomy is more often offered but is associated with high burden of care as well as short- and long-term morbidity and mortality. ${ }^{30-32}$ Most people with ALS decline this alternative. ${ }^{26}$

Full-time NIV using different interfaces has been reported, including in ALS. Bach et al ${ }^{18}$ reported NIV via the mouth in 257 subjects with neuromuscular diseases (5 with ALS), of whom 144 used 24-h NIV (2 with ALS). With adequate bulbar muscle function, mouthpiece ventilation was shown to be an effective alternative to tracheostomy, optimizing convenience and communication. Mouthpiece ventilation was also shown to be a critical component in the weaning of 155 of 157 "unweanable" subjects with neuromuscular diseases. ${ }^{19}$

One study assessed variables associated with successful use of full-time NIV in 27 subjects with ALS and $<15$ min of ventilator-free breathing time (10 used mouthpiece ventilation). The ability to produce assisted peak cough flow $>180 \mathrm{~L} / \mathrm{min}$ and to generate a maximum insufflation capacity-VC difference was associated with prolonged survival without tracheostomy, independent of VC. ${ }^{17}$ Both of these predictive factors correlate with oropharyngeal and laryngeal muscle function. ${ }^{23}$ In addition, studies have shown intolerance to NIV more frequently in subjects with bulbar impairment. 4,6,8,11

Our study is consistent with these data. All but one subject successful with mouthpiece ventilation demonstrated a maximum insufflation capacity-VC difference. Seven subjects had peak cough flow with lung-volume 


\section{Daytime Mouthpiece NIV in Subjects With ALS}

recruitment $<180 \mathrm{~L} / \mathrm{min}$ at the initiation of mouthpiece ventilation; although succeeding with mouthpiece ventilation, time to tracheostomy or death was significantly less than for subjects reaching peak cough flow with lungvolume recruitment $>180 \mathrm{~L} / \mathrm{min}$. Adequate peak cough flow and airway clearance are critical in preventing pneumonia and respiratory failure. The constant availability of mouthpiece ventilation allows frequent lung-volume recruitment, airway clearance, and possibly maintenance of respiratory compliance. Of the 4 subjects who failed to use mouthpiece ventilation successfully, only one was able to show a maximum insufflation capacity-VC difference, only one had a peak cough flow $>180 \mathrm{~L} / \mathrm{min}$, and none was able to increase peak cough flow with lung-volume recruitment.

The b-ALSFRS-R score discriminated between those subjects having sufficient bulbar function to succeed with mouthpiece ventilation $(\geq 6)$ and those who did not $(0-3)$. Utilization of this score has been described in one study assessing swallowing by oropharyngo-esophageal scintigraphy. ${ }^{24}$ Further studies are needed to define its role, but it is easily obtained and clearly useful.

The only randomized controlled trial of NIV in individuals with ALS demonstrated improvement of survival in all subjects and in the subgroup with good bulbar function (216 d [range 94-681 d]). ${ }^{3}$ The survival from NIV initiation until tracheostomy or death of our subjects with preserved bulbar function using mouthpiece ventilation compares favorably, with a median of $648 \mathrm{~d}$ (range 176$2,188 \mathrm{~d}$ ). Our more inclusive criteria for NIV initiation may have led to earlier introduction of NIV, which could partly explain improved survival. However, survival from mouthpiece ventilation initiation to tracheostomy or death is still higher, with a median of $286 \mathrm{~d}$ (range 41-1,769d). Aboussouan et $\mathrm{al}^{6}$ reported a median survival of 15 months ( $450 \mathrm{~d}$ ) for 18 subjects tolerant to NIV introduced because of new orthopnea, new hypercapnia, or both. In a retrospective study by Kleopa et al, ${ }^{12} \mathrm{NIV}$ was introduced when FVC was $<50 \%$ of predicted; the 38 adherent subjects had an average survival from introduction of NIV of $14.2 \pm 13.0$ months $(426 \pm 390 \mathrm{~d})$. The mean survival of our subjects from introduction of NIV was $669 \pm 473 \mathrm{~d}$. More recently, Berlowitz et al ${ }^{13}$ reported a median survival from symptom onset of 32 months (960 d) in NIV users, whereas our subjects using mouthpiece ventilation survived a median of 1,353 d after symptom onset.

As is the case with the initiation of nocturnal NIV in ALS, demonstration of daytime hypercapnia, although present in 5 of 37 subjects, was not a requirement for the introduction of mouthpiece ventilation. NIV is capable of prolonging the survival of individuals suffering from ALS but is also of important palliative value. As such, the addition of mouthpiece ventilation was based primarily on the objective of improving quality of life related to pro- longed hours of inconvenient and uncomfortable mask use, $>12$ h daily ( 24 of 37 subjects), and symptoms of dyspnea (9 of 37 subjects).

Studies evaluating respiratory status in ALS reported that FVC, expressed as a percentage of predicted value (FVC\%), at diagnosis and its rate of decline were the most relevant prognostic factors of pulmonary function assessment. ${ }^{33-35} \mathrm{FVC} \%$ was not related to time to tracheostomy or death in our population successful with mouthpiece ventilation. Given that the ability to generate a maximum insufflation capacity-FVC difference and reach peak cough flow with lung-volume recruitment $>180 \mathrm{~L} / \mathrm{min}$ is related to successful continuous NIV and mouthpiece ventilation, these capacities appear to be more powerful predictors of survival than FVC\%.

Our study is limited because of its retrospective nature and missing data. It was not designed to establish formal successful predictive factors that will require prospective study. Although this is clearly a selected population, approximately $15 \%$ of ALS individuals in our care, those with preserved bulbar function may benefit from the introduction of mouthpiece ventilation rather than the placement of a tracheostomy for an arbitrary number of hours of daily NIV use or continuous use of an NIV mask. We were, however, unable to compare the survival of our subjects using mouthpiece ventilation with a similar population using 24-h mask ventilation because such individuals are very uncommon in our practice and may not return to the clinic for repeated evaluations. Since airway clearance is critical to survival, it would have been helpful to have data on actual use of lung-volume recruitment or other methods to determine its impact, but these data were not consistently available, and few subjects had access to mechanical cough assistance.

\section{Conclusions}

Mouthpiece ventilation is a safe, convenient, and effective way to provide NIV in individuals with ALS requiring continuous ventilatory support and maintaining adequate bulbar function. Mouthpiece ventilation should be offered as an alternative to tracheostomy for individuals able to hold a mouthpiece, protect the airway, and assist cough flows for airway clearance. The b-ALFSRFS-R score seems to be a simple and useful tool to assess candidacy for mouthpiece ventilation.

\section{ACKNOWLEDGMENTS}

We thank Bill Petrcich (Ottawa Hospital Research Institute) for assistance with statistical analysis.

\section{REFERENCES}

1. Corcia P, Pradat PF, Salachas F, Bruneteau G, Forestier NI, Seilhean $\mathrm{D}$, et al. Causes of death in a post-mortem series of ALS patients. Amyotroph Lateral Scler 2008;9(1):59-62. 


\section{Daytime Mouthpiece NIV in Subjects With ALS}

2. Haverkamp LJ, Appel V, Appel SH. Natural history of amyotrophic lateral sclerosis in a database population: validation of a scoring system and a model for survival prediction. Brain 1995;118(Pt 3): 707-719.

3. Bourke SC, Tomlinson M, Williams TL, Bullock RE, Shaw PJ, Gibson GJ. Effects of non-invasive ventilation on survival and quality of life in patients with amyotrophic lateral sclerosis: a randomized controlled trial. Lancet Neurol 2006;5(2):140-147.

4. Ferrero E, Prats E, Povedano M, Martinez-Matos, Manresa F, Escarrabill J. Survival in ALS with home mechanical ventilation: the impact of systematic respiratory assessment and bulbar involvement. Chest 2005;127(6):2132-2138.

5. Pinto AC, Evangelista T, Carvalho M, Alves MA, Sales Luís ML. Respiratory assistance with a noninvasive ventilator (Bipap) in MND/ ALS patients: survival rates in a controlled trial. J Neurol Sci 1995; 129(Suppl):19-26.

6. Aboussouan LS, Khan SU, Meeker DP, Stelmach K, Mitsumoto H. Effect of noninvasive positive-pressure ventilation on survival in amyotrophic lateral sclerosis. Ann Intern Med 1997;127(6):450-453.

7. Newsom-Davis IC, Lyall RA, Leigh PN, Moxham J, Goldstein LH. The effect of noninvasive positive pressure ventilation on cognitive function in amyotrophic lateral sclerosis (ALS): a prospective study. J Neurol Neurosurg Psychiatry 2001;71(4):482-487.

8. Aboussouan LS, Khan SU, Banerjee M, Arroliga AC, Mitsumoto H. Objective measures of the efficacy of noninvasive positive pressure ventilation in amyotrophic lateral sclerosis. Muscle Nerve 2001; 24(3):403-409.

9. Lyall RA, Donaldson N, Fleming T, Wood C, Newsom-Davis I, Polkey MI, et al. A prospective study of quality of life in ALS patients treated with noninvasive ventilation. Neurology 2001;57(1):153-156.

10. Bourke SC, Bullock RE, Williams TL, Shaw PJ, Gibson GJ. Noninvasive ventilation in ALS: indications and effect on quality of life. Neurology 2003;61(2):171-177.

11. Lo Coco D, Marchese S, Pesco MC, La Bella V, Piccoli F, Lo Coco A. Noninvasive positive-pressure ventilation in ALS: predictors of tolerance and survival. Neurology 2006;67(5):761-765.

12. Kleopa KA, Sherman M, Neal B, Romano GJ, Heiman-Patterson T. Bipap improves survival and rate of pulmonary function decline in patients with ALS. J Neurol Sci 1999;164(1):82-88.

13. Berlowitz DJ, Detering K, Schachter L. A retrospective analysis of sleep quality and survival with domiciliary ventilatory support in motor neuron disease. Amyothroph Lateral Scler 2006;7(2):100-106.

14. Bach JR. Amyotrophic lateral sclerosis: prolongation of life by noninvasive respiratory AIDS. Chest 2002;122(1):92-98.

15. Lechtzin N, Scott Y, Busse AM, Clawson LL, Kimball R, Wiener $\mathrm{CM}$. Early use of noninvasive ventilation prolongs survival in subjects with ALS. Amyotroph Lateral Scler 2007;8(3):185-188.

16. Benditt JO. Full-time noninvasive ventilation: possible and desirable. Respir Care 2006;51(9):1005-1012; discussion 1012-1015.

17. Bach JR. Amyotrophic lateral sclerosis: predictors for prolongation of life by noninvasive respiratory aids. Arch Phys Med Rehabil 1995;76(9):828-832.

18. Bach JR, Alba AS, Saporito LR. Intermittent positive pressure ventilation via the mouth as an alternative to tracheostomy for 257 ventilator users. Chest 1993;103(1):174-182.
19. Bach JR, Gonçalves MR, Hamdani I, Winck JC. Extubation of patients with neuromuscular weakness: a new management paradigm. Chest 2010;137(5):1033-1039.

20. Brooks BR, Miller RG, Swash M, Munsat TL, World Federation of Neurology Research Group on Motor Neuron Diseases. El Escorial revisited: revised criteria for the diagnosis of amyotrophic lateral sclerosis. Amyotroph Lateral Scler Other Motor Neuron Disord 2000; 1(5):293-299.

21. Hankinson JL, Odencrantz JR, Fedan KB. Spirometric reference values from a sample of the general U.S. population. Am J Respir Crit Care Med 1999;159(1):179-187.

22. Kang SW, Bach JR. Maximum insufflation capacity. Chest 2000; 118(1):61-65.

23. Cedarbaum JM, Stambler N, Malta E, Fuller C, Hilt D, Thurmond B, Nakanishi A. The ALSFRS-R: a revised ALS functional rating scale that incorporates assessments of respiratory function. BDNF ALS Study Group (Phase III). J Neurol Sci 1999;169(1):13-21.

24. Fattori B, Grosso M, Bongioanni P, Nacci A, Cristofani R, AlSharif A, et al. Assessment of swallowing by oropharyngoesophagealscintigraphy in patients with amyotrophic lateral sclerosis. Dysphagia 2006;21(4):280-286.

25. Bach JR. Mechanical insufflation-exsufflation: comparison of peak expiratory flows with manually assisted and unassisted coughing techniques. Chest 1993;104(5):1553-1562.

26. McKim DA, King J, Walker K, Leblanc C, Timpson D, Wilson KG, et al. Formal ventilation patient education for ALS predicts real-life choices. Amyotroph Lateral Scler 2012;13(1):59-65.

27. Miller RG, Jackson CE, Kasarskis EJ, England JD, Forshew D, Johnston W, et al. Practice parameter update: the care of the patient with amyotrophic lateral sclerosis: drug, nutritional, and respiratory therapies (an evidence-based review). Neurology 2009;73(15):12181226.

28. McKim DA, Road J, Avendano M, Abdool S, Cote F, Duguid N, et al. Home mechanical ventilation: a Canadian Thoracic Society clinical practice guideline. Can Respir J 2011;18(4):197-215.

29. Sandoz JS, LeBlanc C, McKim DA. Data downloads for effective non-invasive ventilation in neuromuscular respiratory failure. Respir Care 2014;59(3):e35-e40.

30. Dhand R, Johnson JC. Care of the chronic tracheostomy. Respir Care 2006;51(9):984-1004; discussion 1002-1004.

31. Durbin CG. Early complications of tracheostomy. Respir Care 2005; 50(4):511-515

32. Epstein SK. Late complications of tracheostomy. Respir Care 2005; 50(4):542-549.

33. Stambler N, Charatan M, Cedarbaum JM. Prognostic indicators of survival in ALS: ALS CNTF Treatment Study Group. Neurology 1998;50(1):66-72.

34. Czaplinski A, Yen AA, Appel SH. Forced vital capacity (FVC) as an indicator of survival and disease progression in an ALS clinic population. J Neurol Neurosurg Psychiatry 2006;77(3):390-392.

35. Ringel SP, Murphy JR, Alderson MK, Bryan W, England JD, Miller RG, et al. The natural history of amyotrophic lateralsclerosis. Neurology 1993;43(7):1316-1322. 